\title{
Tangence
}

\section{Deconstructing Woody Allen}

\section{Chantal Pouliot}

Numéro 68, hiver 2002

\section{Littérature et mathématiques}

URI : https://id.erudit.org/iderudit/008247ar

DOI : https://doi.org/10.7202/008247ar

Aller au sommaire du numéro

Éditeur(s)

Presses de l'Université du Québec

ISSN

0226-9554 (imprimé)

1710-0305 (numérique)

Découvrir la revue

Citer cet article

Pouliot, C. (2002). Deconstructing Woody Allen. Tangence, (68), 51-64.

https://doi.org/10.7202/008247ar
Résumé de l'article

Cet article propose un examen théorique des jeux de fiction dans Deconstructing Harry de Woody Allen. S'appuyant sur les travaux d'Umberto Eco, de Lubomir Dolezel et de Marie-Laure Ryan, l'analyse se concentre sur les problèmes posés par les identités multiples des personnages ainsi que sur les processus d'autoréférence, de fragmentation et de récursivité. d'utilisation que vous pouvez consulter en ligne.

https://apropos.erudit.org/fr/usagers/politique-dutilisation/ 


\title{
Deconstructing Woody Allen
}

\author{
Chantal Pouliot, Université Laval
}

\begin{abstract}
Cet article propose un examen théorique des jeux de fiction dans Deconstructing Harry de Woody Allen. S'appuyant sur les travaux d'Umberto Eco, de Lubomir Dolezel et de Marie-Laure Ryan, l'analyse se concentre sur les problèmes posés par les identités multiples des personnages ainsi que sur les processus d'autoréférence, de fragmentation et de récursivité.
\end{abstract}

\section{La fiction et Woody Allen}

Dans Deconstructing Harry, Woody Allen propose un univers étrange où la fiction et la réalité ${ }^{1}$ ne sont pas mutuellement exclusives. Le réel contamine la fiction et la fiction s'immisce indubitablement dans le réel, avec sa logique et ses valeurs de vérité. Par le biais des dialogues, par les situations engendrées, par le rythme, le chevauchement et la confrontation de Harry Block avec lui-même, la fiction fait sans cesse l'objet d'un regard critique. Deconstructing Harry poursuit, accentue et complexifie une démarche métafictionnelle déjà présente dans The Purple Rose of Cairo (1985) et dans "The Kugelmas Episode ${ }^{2}$ ». C'est à une crise de l'identité réelle et fictive qu'Harry Block et ses différents doubles se butent. On assiste à une déconstruction du personnage ainsi qu'à une fragmentation des identités dans chacune des micro-fictions nées de la plume de Block. Une accumulation de récits, de paroles, d'entrecroisements de discours et d'expériences confère ainsi au film une richesse théorique certaine.

L'analyse que je propose de la fiction dans Deconstructing Harry prend appui sur le scénario qui permettra d'étayer le propos par des citations. Il est à noter que les résultats, compte tenu de la perspective adoptée, s'appliqueraient au film lui-même. En effet, le film, comme le scénario, en plus d'être traversé par l'humour,

1. Par «réalité», je fais ici référence à la réalité fictive, c'est-à-dire celle du personnage principal, Harry, et non à celle du lecteur.

2. Woody Allen, "The Kugelmas Episode», dans Side Effects, New York, Random House, 1980, p. 41-45. 
porte la marque des identités multiples, de l'auto-référence, de la fragmentation et, finalement, de la récursivité. À ce propos, Antoine de Baecque, dans son introduction au scénario de Deconstructing Harry, mentionne que

le montage du film est proprement sidérant, à déconseiller absolument dans toute école de cinéma: les faux raccords foisonnent, les images se bousculent les unes les autres, se chevauchent, tourbillon où les collures entre les plans ont l'air aussi importantes que les plans eux-mêmes ${ }^{3}$.

Ici, je m'attarderai surtout à la problématique de la fictionnalité, plus spécifiquement aux modalités régissant les différents niveaux de fiction, à la remise en question de leur hiérarchie, à la dépendance des niveaux entre eux et, finalement, à l'enchâssement des univers dans le scénario. À cette fin, je proposerai deux modèles: un modèle séquentiel illustrant la succession des événements et un modèle structural de l'organisation des différents niveaux fictifs. La démarche s'appuie sur des textes liés à la sémantique des mondes possibles qui, elle-même, trouve son fondement dans la théorie des ensembles en mathématiques ${ }^{4}$.

\section{La construction des mondes fictionnels}

Avant d'aborder l'étude de Deconstructing Harry, il n'est sans doute pas inutile de s'attarder un peu à cette sémantique des mondes possibles afin de préciser les bases théoriques de l'analyse. La sémantique des mondes possibles est une théorie sémantique moderne qui s'inspire de la philosophie classique, notamment de l'idée, chère à Leibniz, d'une infinité de mondes possibles ${ }^{5}$. Cette théorie a l'avantage d'offrir une solution au problème de la

3. Woody Allen, Deconstructing Harry/Harry dans tous ces états, dialogues traduits de l'américain par Jacqueline Cohen, Paris, Cahiers du cinéma, 1998, p. 7. Désormais, les références à cet ouvrage seront indiquées par le sigle $D H$, suivi de la page, et placées entre parenthèses dans le corps du texte.

4. Voir ici deux textes d'Umberto Eco: «Petits mondes", dans Les limites de l'interprétation, traduit de l'italien par Myriem Bouzaher, Paris, Le livre de poche, coll. «Biblio essai», 1992, p. 212-233, de même que «Structures de mondes", dans Lector in fabula. La coopération interprétative dans les textes narratifs, traduit de l'italien par Myriem Bouzaher, Paris, Grasset, coll. «Figures», 1985, p. 160-230. Voir également Lubomir Dolezel, «Pour une typologie des mondes fictionnels ", dans Hermann Parret et Hans-George Ruprecht (sous la dir. de), Exigences et perspectives de la sémiotique. Recueil d'hommages pour Algirdas-Julien Greimas, Amsterdam, John Benjamins, 1985, t. 1, p. 7-23.

5. Voir ses Essais de théodicée (1710). 
référence fictive, en plus de fournir des modèles relevant de la théorie des ensembles utilisés en logique formelle ${ }^{6}$.

Les théories les plus connues de la référence fictionnelle sont partagées entre la thèse de son inexistence (on parle alors de nonréférence: les textes ne réfèrent pas) et celle de l'auto-référence (la fiction ne réfère qu'à elle-même). La sémantique des mondes possibles offre sans doute une avenue plus féconde en proposant que la fiction réfère à une multitude de mondes possibles construits par les textes: "Les mondes fictionnels ne préexistent pas aux textes; de sorte que tout texte littéraire doit construire son propre domaine de référence, et doit faire exister son propre monde fictionnel ${ }^{7}$ ». Ainsi, la fonction référentielle vise des mondes fictionnels possibles plutôt que le monde réel (ou actuel) ${ }^{8}$.

Si les mondes possibles (ensembles dans lesquels on retrouve des agents, des actions, etc.) doivent être vus comme des constructions culturelles et si l'on veut confronter les mondes, leurs valeurs et leurs propriétés, notre monde réel $\mathrm{W}_{\mathrm{L}}$ (monde des lecteurs que nous sommes tous) doit être considéré comme une construction culturelle. D'ailleurs, la théorie considère l'ensemble des images du monde actuel comme une encyclopédie potentiellement complète, référence d'un univers présentant des propriétés et soumis à des forces et à des contraintes naturelles ${ }^{9}$.

On peut ainsi élaborer - Lubomir Dolezel s'y est employé une taxinomie permettant d'introduire un certain ordre dans les concepts liés à la construction de mondes fictifs. Les structures, les formes et les modes d'existence des mondes fictionnels sont déterminés par des procédés de construction. Ces procédés, qu'il nomme macro-contraintes, sont de deux ordres (extensionnels et intensionnels) et forment un système ouvert qu'on pourra certainement enrichir avec le temps.

Les macro-contraintes intensionnelles sont des constituants non référentiels du sens du texte, alors que les extensions s’identifient à la

6. Saul A. Kripke, "Semantical Considerations on Modal Logic», Acta Philosophica Fennica, Helsinki, XVI, 1963, p. 83-94, et Jaakko Hintikka, The Intentions of Intentionality and Other New Models for Modalities, Dordrecht, Reidel, 1975.

7. Lubomir Dolezel, "Pour une typologie des mondes fictionnels », art. cité, p. 9.

8. Dans le cadre d'une approche constructiviste des mondes possibles, le monde «réel» de référence, en l'occurrence celui du lecteur, doit être entendu comme une construction culturelle.

9. Cette dernière remarque est tirée d'Umberto Eco, "Structures de mondes", art. cité, p. 173. 
relation référentielle. Dans l'analyse de Deconstructing Harry, je m'attarderai aux macro-contraintes extensionnelles, la problématique de la référence de la fiction dans le scénario de Woody Allen étant particulièrement importante. Plus précisément, je m'attacherai à ce sous-ensemble des macro-contraintes extensionnelles que sont les macro-contraintes modales. Il s'agit de macro-règles formatives essentielles dans la construction des mondes fictionnels et qui ont pour rôle (entre autres) de déterminer le potentiel narratif de ces mondes. Le système des modalités narratives s'inspire des études en logique sémantique moderne et notamment des travaux de von Wright. On suppose alors que les mondes fictionnels mettent en jeu quatre types de modalités:

1) les modalités aléthiques sont des contraintes portant sur la possibilité, l'impossibilité et la nécessité de propriétés, d'états de choses et d'événements dans un monde fictionnel donné;

2) les modalités épistémiques sont des opérateurs du savoir, de l'ignorance et du croire;

3) les modalités déontiques réfèrent à la permission, à l'interdiction et à l'obligation;

4) les modalités axiologiques convoquent les notions de bien, de mal et d'amoralité.

\section{Deconstructing Harry}

Dans Deconstructing Harry, Woody Allen met en scène le personnage de Harry Block, un auteur d'écrits autobiographiques névrosé, tourmenté, s'apitoyant sans cesse sur lui-même. Le film est constitué de courtes séquences hétérogènes. Woody Allen (le cinéaste-créateur) insère, entre les séquences de la réalité actuelle de Block (qu'on nommera l'univers actuel de Harry), une ou des séquences extraites des fictions romanesques autobiographiques écrites par Block, des flash-back de sa vie ou même les fantasmes qu'il entretient. Deconstructing Harry, malgré une fluidité et une cohérence surprenantes, nécessite un important travail intellectuel de spectature (comme on dit: de lecture), ne serait-ce que pour reconstruire l'histoire (il serait plus juste de dire les histoires).

Avant de vérifier si les mondes réel et fictif sont régis par les mêmes macro-contraintes extensionnelles modales, établissons la structure hiérarchique des niveaux de fictionnalité. Cette étape est 
primordiale car c'est sur ces données de base que reposera, entre autres, l'interprétation du phénomène de contamination de la réalité fictive par le fictif romanesque.

\section{Niveaux de fictionnalité}

Le film de Woody Allen, donc, est ponctué de séquences fictives (fictions dans la fiction) qui sont des versions cinématographiques des extraits romanesques hautement autobiographiques écrits par Harry Block. Je propose de considérer l'univers de Harry Block comme le premier niveau fictionnel et le symboliserai par $\mathrm{W}_{\mathrm{H}}$ (conformément à la typologie qu'Umberto Eco emploie notamment dans «Structures de mondes»). Ainsi, les mondes fictifs romanesques construits par Block constituent le deuxième niveau de la fiction ${ }^{10}$. On retrouve à ce niveau trois mondes principaux différents (on ne sait pas s'ils proviennent de romans distincts, mais on suppose que oui puisque les personnages, qui correspondent à des étapes différentes de la vie de Harry, ont des prénoms différents). Les mondes du deuxième niveau seront nommés

$$
\begin{aligned}
\mathrm{W}_{\mathrm{KJ}}= & \text { Ken et Janet }\left(3^{\mathrm{e}} \text { mariage } \text {;ane }\right) \\
\mathrm{W}_{\mathrm{PH}}= & \begin{array}{l}
\text { Paul et Helen }\left(2^{\mathrm{e}} \text { mariage }\right. \text {; contreparties de Harry et } \\
\text { Joan })
\end{array} \\
\mathrm{W}_{\mathrm{HR}}= & \begin{array}{l}
\text { Harvey et Rosalee }\left(1^{\mathrm{er}} \text { mariage }\right. \text {; contreparties de } \\
\text { Harry et d'un personnage indéterminé })
\end{array}
\end{aligned}
$$

Le scénario contient aussi des flash-back de la vie réelle de Harry Block qui ont souvent une fonction explicative-justificatrice ainsi que des fantasmes qui, pour simplifier l'analyse (et l'élaboration du modèle structural), seront classés au niveau premier de fictionnalité puisqu'ils font partie de l'univers réel actuel de Harry ${ }^{11}$.

10. Soulignons que ces mondes fictifs au deuxième degré sont des contreparties de la vie de Block. La notion de contrepartie sert à désigner les versions d'une entité (personnages, lieu, etc.) appartenant à différents mondes possibles (voir David Lewis, "Counterpart Theory and Quantified Modal Logic», dans The Journal of Philosophy, New York, vol. LXV, no 5, 1968, p. 113-126). Cette prémisse est primordiale pour la poursuite de la lecture.

11. On pourrait qualifier ces mondes de mondes possibles indépendants de $\mathrm{W}_{\mathrm{H}}$ puisque les propriétés qu'on retrouve dans les rêves et fantasmes sont différents de $\mathrm{W}_{\mathrm{H}}$. Mais accordons-nous pour dire que tous ces mondes possibles sont des sous-ensembles faisant partie du même niveau (le premier). 


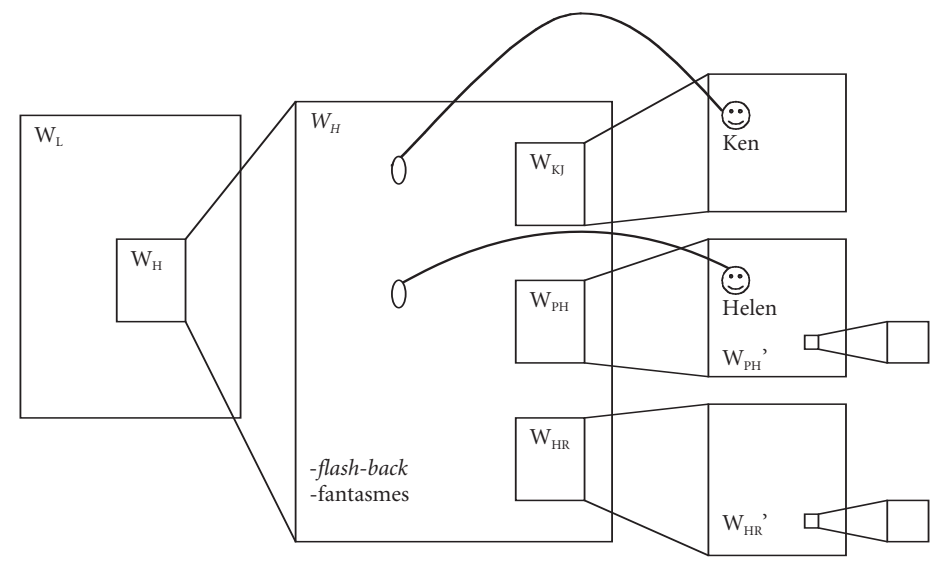

$$
\begin{array}{ll}
\text { Niveaux } & \text { Univers ou Mondes possibles } \\
1 & \mathrm{~W}_{\mathrm{H}}, \\
2 & \mathrm{~W}_{\mathrm{K}}, \mathrm{W}_{\mathrm{PH}}, \mathrm{W}_{\mathrm{HR}} \\
3 & \mathrm{~W}_{\mathrm{PH}}, \mathrm{W}_{\mathrm{HR}}
\end{array}
$$

Modèle structural des mondes possibles enchâssés dans Deconstructing Harry de Woody Allen

Un troisième (et dernier) niveau de fictionnalité est constitué des mondes créés par les personnages romanesques inventés par Block. On sait de Paul et Harvey (deux contreparties de Harry) qu'ils sont des écrivains ${ }^{12}$, même si les indices que nous avons sur les mondes qu'ils construisent sont minces:

Intérieur nuit. Appartement de Harry et Rosalee.

Début de la séquence de fiction.

Harvey Stern, un jeune écrivain, tape laborieusement à la machine avec un seul doigt.

HARRY (off): Si Harvey Stern s'était marié trop jeune, c'était surtout pour fuir l'appartement de ses parents... [...] Le jour il travaillait mollement dans un magasin de chaussures. La nuit, il se penchait avec une intense exubérance sur sa Remington portative.

12. Ceci est un cas éloquent de récursivité de la fiction autobiographique: si Harry Block se projette dans ses personnages, ceux-ci, comme lui, devront être écrivains et se projeter à leur tour dans leurs personnages respectifs qui, eux-mêmes, etc. 
Intérieur jour. Appartement de Paul et Helen.

Paul tape à la machine.

HARRY (voix off, récit): "Ils vécurent deux années extraordinaires. Ils s'installèrent dans un grand appartement sur West End Avenue. Il écrivait... Elle recevait ses patients. Mais, ce qu'elle analysait mieux que tout, c'était l'œuvre de son mari» (DH, p. 63).

À première vue, le lecteur du scénario (ou le spectateur du film) pourrait penser que les univers construits par Harry Block $\left(\mathrm{W}_{\mathrm{KJ}}, \mathrm{W}_{\mathrm{PH}}, \mathrm{W}_{\mathrm{HR}}\right)$ sont régis par les mêmes macro-contraintes extensionnelles ${ }^{13}$ que $W_{H}$ puisque Block écrit des romans autobiographiques à fortes tendances réalistes. Toutefois, une lecture attentive permet de remarquer des différences majeures et fondamentales au niveau de certaines macro-contraintes extensionnelles modales.

\section{Jeux de modalités}

Le premier niveau $\left(\mathrm{W}_{\mathrm{H}}\right)$ et le second niveau $\left(\mathrm{W}_{\mathrm{KJ}}, \mathrm{W}_{\mathrm{PH}}\right.$ et $\mathrm{W}_{\mathrm{HR}}$ ) diffèrent quant à leurs caractéristiques modales. Ainsi, les modalités aléthiques qui régissent les mondes varient selon les niveaux et selon les mondes.

Par exemple, dans une séquence de fiction extraite de la nouvelle Le comédien écrite par Block, le tournage d'un film doit s'interrompre parce que l'acteur principal est flou. À la grande surprise de l'équipe technique et de sa famille, Mel demeure dans cet état pendant quelque vingt-quatre heures. Dans son récit, Block rend possible ce qui est physiologiquement impossible dans $\mathrm{W}_{\mathrm{L}}$ (le monde du lecteur) et, croit-on, dans $\mathrm{W}_{\mathrm{H}}$. Or, vers la fin du scénario, l'on s'aperçoit qu'il est aussi possible dans $\mathrm{W}_{\mathrm{H}}$ d'être flou, puisque Harry est victime de cet inconfort à l'occasion d'un moment d'angoisse. Il en découle un double effet de surprise pour le lecteur (évoluant dans $\mathrm{W}_{\mathrm{L}}$ ) qui croyait jusque-là que l'état de Mel était le fruit de l'imagination débordante de Harry, mais qui s'aperçoit que celui-ci en a puisé l'idée parmi une multitude de possibles réels ${ }^{14}$.

Au début du film (ou de la lecture du scénario), le lecteur suppose ${ }^{15}$ que $\mathrm{W}_{\mathrm{H}}$ correspond en grande partie à son propre système

13. Dont on a précédemment mentionné les quatre types de modalités constitutives: aléthiques, épistémiques, déontiques et axiologiques.

14. On pourrait aussi supposer que le niveau narratif dans lequel se trouve Mel contamine $\mathrm{W}_{\mathrm{H}}$. En ce cas, les modalités aléthiques qui régissent les relations entre les mondes fictifs sont elles-mêmes affectées. Nous verrons plus loin que d'autres éléments du scénario tendent à accréditer cette hypothèse. 
référentiel ${ }^{16}$, à son encyclopédie culturelle. Effectivement, à première vue, le monde $\mathrm{W}_{\mathrm{H}}$ semble soumis aux mêmes modalités aléthiques que le monde $\mathrm{W}_{\mathrm{L}}$. Le lecteur réalise son erreur et ajuste sa conception de $\mathrm{W}_{\mathrm{H}}$ lors de deux séquences particulièrement intéressantes sur le plan de la conjonction des mondes fictifs réels et fictifs romanesques.

Dans ces séquences, les niveaux enchâssés de la fiction contaminent le réel $\left(\mathrm{W}_{\mathrm{H}}\right)$. La rencontre de Harry Block et de certains des personnages qu'il a créés (Ken et Helen) démontre l'hybridité aléthique de $\mathrm{W}_{\mathrm{H}}$, c'est-à-dire qu'il n'existe plus de frontière entre les domaines naturels et surnaturels; ceux-ci coexistent et s'entrecroisent. La contamination du réel de Harry Block par un niveau supérieur de la fiction constitue donc un événement important sur le plan aléthique, plus spécifiquement sur le plan des contraintes de possibilité et d'impossibilité, puisque le monde $\mathrm{W}_{\mathrm{L}}$ (du lecteur) n'est pas un monde hybride (c'est-à-dire dans lequel les phénomènes naturels et surnaturels s'entrecroisent), mais un monde où, semble-t-il, le domaine du naturel et celui du surnaturel sont distincts, voire étanches.

La contamination du réel produite par l'intrusion des personnages romanesques permet l'émergence de phénomènes épistémiques (liés au savoir ou, plus exactement, à l'ignorance). Non seulement le personnage de Ken (la contrepartie de Harry alors qu'il était l'époux de Jane) sait qu'il est la création de Harry, mais il possède une connaissance élargie qui s'étend à l'univers de Block. Autrement dit, Ken est au courant de choses que même son créateur ne connaît pas:

Harry se dirige vers Ken en train de regarder dans le viseur d'un nickelodéon. Ken se redresse.

KEN : Pisse-froid, ta troisième femme. Elle aurait jamais fait une bonne mère.

HARRY: Qui êtes-vous?

KEN : C'est moi, Ken.

HARRY: Ken?

KEN (riant): Écoutez-moi ça! C’est toi qui m'as créé et tu me reconnais pas ${ }^{17}$.

15. Supposition inexacte qui provient de constatations partielles.

16. Ce système est le cadre de référence dont le lecteur dispose avant d'entreprendre la lecture du scénario et indépendamment de celui-ci.

17. Cette remarque joue, entre autres, sur les écrits de Block en regard de leur représentation cinématographique: Block devrait être en mesure de reconnầtre le personnage qu'il a lui-même créé, décrit, défini. 
$[\ldots]$

HARRY: Comment tu sais tout ça, toi?

KEN : Ben, je suis toi. À peine déguisé. Tu m’as donné un peu plus de maturité et un nom différent.

HARRY: Quoi, tu veux dire que je... je sais tout ça?

KEN : Mais bien sûr! [...]

HARRY: Hé, je vais pas rester là à me faire sermonner par ma propre création.

KEN : Oh... moi, tu peux pas me bluffer. Je suis pas comme ton psy. Lui, il connaît que ta version. Moi je sais la vérité! ( $D H$, p. 103)

La hiérarchie des niveaux fictifs ainsi que la distinction entre les mondes $\mathrm{W}_{\mathrm{H}}$ et $\mathrm{W}_{\mathrm{KJ}}$ sont à la fois maintenues (Ken déclare: "C'est toi qui m'a créé») et abolies (Ken s'entretient avec Harry).

De plus, on assiste lors de l'entrecroisement momentané des niveaux premier $\left(\mathrm{W}_{\mathrm{H}}\right)$ et second $\left(\mathrm{W}_{\mathrm{KJ}}\right.$ ou $\left.\mathrm{W}_{\mathrm{PH}}\right)$ à l'émergence de vérités objectives: le lecteur ou spectateur a alors accès à de nouvelles informations concernant $\mathrm{W}_{\mathrm{H}}$ mais auxquelles Harry ne peut accéder, puisqu'il est absent lors du déroulement des séquences en question. Cet accès à des informations essentielles (ou du moins très utiles) à la compréhension de l'histoire n'est rendu "possible ${ }^{18}$ » que par l'entrecroisement de $\mathrm{W}_{\mathrm{H}}$ et $\mathrm{W}_{\text {fictifs }}$, tel qu'il se produit à deux reprises dans Deconstructing Harry.

La deuxième rencontre de $\mathrm{W}_{\mathrm{H}}$ et $\mathrm{W}_{\text {fictifs }}$ déclenche des phénomènes épistémiques qui méritent d'être notés. On constate que le personnage romanesque possède une connaissance et une conscience très grande de sa condition:

Extérieur jour. Station service.

Harry se tient devant la voiture, toujours perdu dans ses pensées.

Hilly se penche par la fenêtre. À la fenêtre se tient Helen.

HELEN : Alors, comme ça, tu t'es vengé de ton ex-femme et de ta sœur et je suis née!

HARRY: Née? Qu'est-ce que tu racontes?

HELEN : De ta plume, de ta fertile imagination... Et ça, pour de l'imagination...

HARRY: Hé... j'ai pas voulu me venger de Joan!

HELEN : Oh, tu as fait de moi une sorte d'hybride de juive hargneuse qui s'en va avec un de ses patients... un orthodoxe, un divorcé venu de Tel Aviv.

18. Bien sûr il n'existe pas de valeur de vérité dont le référent serait situé dans le monde réel du lecteur. Aussi, "possible» n'a une valeur de vérité que si l'on se situe à l'intérieur de Deconstructing Harry, fiction. 
$[\ldots]$

HELEN : Elle t'aime toujours malgré ton évidente condescendance pour son mode de vie.

HARRY : Elle m'aime?

HELEN: Tu veux entendre ce qu'elle dit de toi quand tu n'es pas là? ( $D H$, p. 147)

Il arrive donc qu'un personnage d'un niveau enchâssé ne se contente pas de communiquer avec un personnage d'un niveau enchâssant, mais qu'il aille jusqu'à dévoiler des informations concernant des discussions auxquelles n'ont participé ni le personnage ni Harry Block lui-même. La conséquence de tout ceci est un enchâssement réciproque des mondes. Par exemple, la vérité «objective» qui émergera du contact des deux niveaux de fiction $-\mathrm{W}_{\mathrm{H}}$ dans le cas de Harry, $\mathrm{W}_{\mathrm{PH}}$ dans celui d'Helen - concernera la sœur de Block et son mari. Helen et Harry s'immisceront, en "hommes invisibles", dans une pièce où ils seront témoins d'une conversation. Encore une fois, l'accès à cette information, à cette vérité «objective», ne serait pas possible pour Harry sans Helen. Et, curieusement, l'information obtenue semble fiable (c'est pourquoi nous parlons de "vérité»), même si le processus qui y mène est hautement suspect.

C'est ce jeu sur les macro-contraintes extensionnelles modales, surtout aléthiques et épistémiques, qui permet à Woody Allen d'explorer la fiction, de la critiquer et d'y introduire des paradoxes. Pourtant, même si le monde $\mathrm{W}_{\mathrm{H}}$ autant que les mondes narratifs de deuxième niveau paraissent invraisemblables et peu crédibles aux lecteurs que nous sommes du point de vue de notre expérience actuelle, il existe des similarités sur le plan logique entre la réalité dans $\mathrm{W}_{\mathrm{L}}$ et la réalité fictive dans $\mathrm{W}_{\mathrm{H}}$ (le monde de Harry Block). Par exemple, les modalités axiologiques en vigueur dans $\mathrm{W}_{\mathrm{H}}$ (convoquant les notions de bien, de mal et d'amoralité) rappellent celles de $\mathrm{W}_{\mathrm{L}}$. L'adultère y est condamnable, de sorte que l'on s'y livre de la façon la plus discrète possible.

Malgré cela, on remarque rapidement, à la lecture du scénario, que le dispositif des mondes enchâssés dans Deconstructing Harry ne respecte pas la hiérarchie et la dépendance auxquelles on se serait attendu. La contamination de la réalité actuelle de Harry $\left(\mathrm{W}_{\mathrm{H}}\right)$ par les univers fictifs $\mathrm{W}_{\mathrm{PH}}$ et $\mathrm{W}_{\mathrm{KJ}}$ est d'autant plus intéressante qu'elle constitue un phénomène d'auto-référence problématique autour de laquelle s'articule le film de Woody Allen. 


\section{Auto-référence et paradoxes}

On remarque, en observant le modèle séquentiel, que malgré l'hétérogénéité des séquences (c'est-à-dire le fait qu'elles concernent une multitude de mondes possibles), Deconstructing Harry est d'une fluidité étonnante. Le rôle que joue l'auto-référence consiste ici à suggérer un fil conducteur qui court à travers les séquences et les mondes possibles. Il semble que Woody Allen se mette en scène ${ }^{19}$ en s'incarnant en Harry Block et que celui-ci se mette à son tour en scène, récursivement, dans ses romans autobiographiques. Un exemple des plus frappants est cet extrait dans lequel il y a alternance entre Harry et ses contreparties du second niveau de fiction. Les personnages changent d'une séquence à l'autre, mais les décors restent les mêmes, indépendamment des niveaux:

Intérieur nuit. Appartement de Ken. Cuisine.

Début de la séquence de fiction.

Ken est au téléphone, très nerveux, constamment anxieux à l'idée que sa femme puisse entrer dans la pièce.

KEN (au téléphone) : Elle entend pas.

LESLIE (off): Je suis en ville.

Intérieur nuit. Chambre d'hôtel de Leslie.

LESLIE (au téléphone) : Norman m'emmène voir Les Misérables mais j'ai une heure devant moi.

Intérieur nuit. Appartement de Ken. Cuisine.

$\mathrm{KEN}$ : Je... je, je sais pas si je pourrai.

Intérieur nuit. Chambre d'hôtel de Leslie.

LESLIE: J'ai réservé notre chambre au Wyndham. Tu sais que ça fait presque deux semaines.

[...]

Intérieur nuit. Appartement de Janet et Ken. Cuisine.

Ken raccroche le téléphone.

KEN (à Janet) : Je vais aller faire un tour.

JANET (étonnée) : Maintenant?

Fin de la séquence de fiction.

19. Antoine de Baecque écrit, dans son introduction: «On ne devrait plus avoir à présenter Woody Allen. Au fil des films, près d'une trentaine à ce jour, le personnage s'est suffisamment incarné à l'écran [...] Qu'il soit Virgil, Alvy, Isaac, Danny, Gaber, ou Larry, Woody Allen est toujours le même. Et pourtant sans cesse différent. [...] Lorsque Woody s'est mué en Harry, à l'occasion de son dernier film, il semble qu'il ait voulu mettre toutes ces identités différentes en un seul personnage. Harry, écrivain new-yorkais, est donc un nouveau Woody de plus» (DH, p. 5). 


$$
\begin{aligned}
& \text { Légende : } \mathrm{W}_{\mathrm{KJ}}=\text { Ken et Janet } \quad\left(3^{\mathrm{e}} \text { mariage ; Harry et Jane }\right) \\
& \mathrm{W}_{\mathrm{PH}}=\text { Paul et Helen } \quad\left(2^{\mathrm{e}} \text { mariage; Harry et Joan }\right) \\
& \mathrm{W}_{\mathrm{HR}}=\text { Harvey et Rosalee } \quad\left(1^{\text {er }} \text { mariage }\right) \\
& \mathrm{W}_{\mathrm{G}}=\text { Goldberg } \quad\left(4^{\mathrm{e}}\right. \text { relation amoureuse) } \\
& \mathrm{W}_{\mathrm{M}}=\mathrm{Mel} \text { (acteur flou) (Le comédien) } \\
& \mathrm{W}_{\mathrm{MD}}=\text { Max et Dolly } \quad \text { (analogues de Burt et Doris) } \\
& \mathrm{W}_{\mathrm{R}}=\text { Rifkin (Harry) }
\end{aligned}
$$

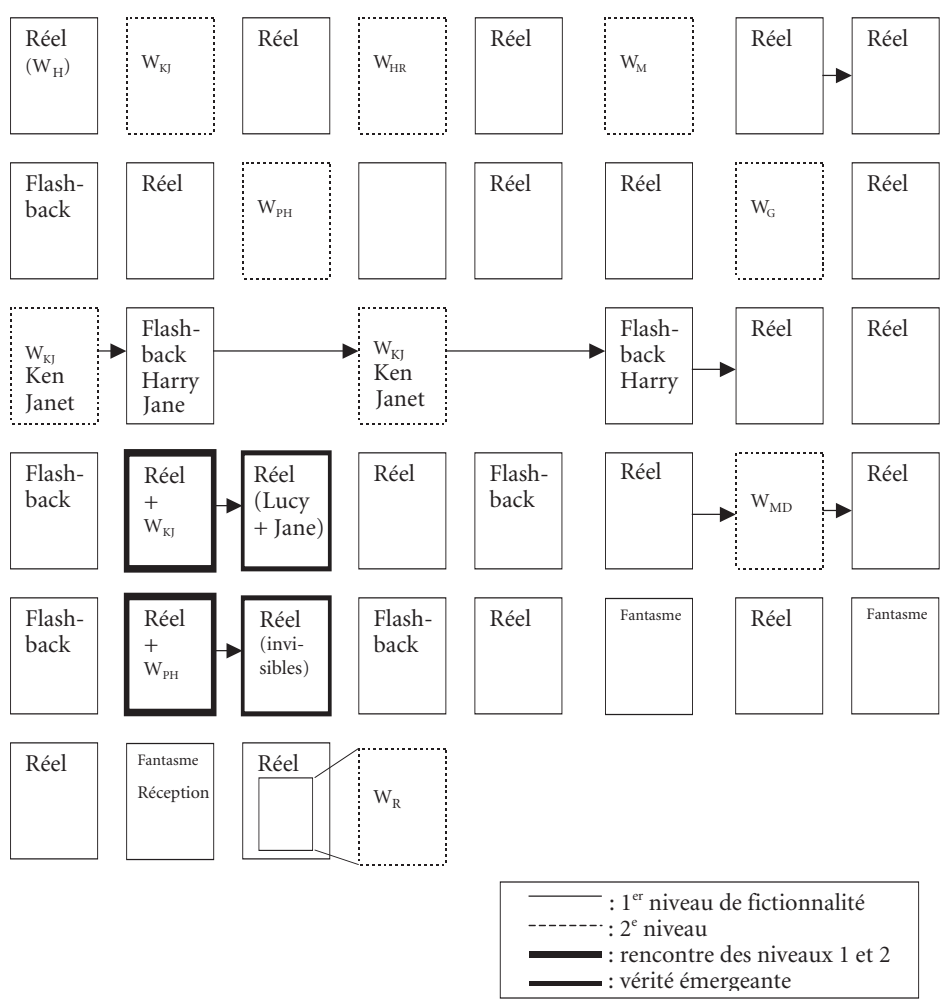

Modèle séquentiel de Deconstructing Harry de Woody Allen

Intérieur nuit. Appartement de Harry et Jane. Cuisine.

Début du flash-back.

La séquence se poursuit, mais en flash-back. C'est le même appartement, mais c'est Harry et sa femme Jane qui s'y trouvent.

HARRY: Oh, oui j'ai envie de... je veux juste... Enfin, j'ai envie de... prendre un peu l'air.

JANE (consciente du mensonge) : Ah! D'accord. 
Extérieur nuit. Rue.

Harry sort de chez lui, court et hèle un taxi.

Intérieur nuit. Hôtel. Chambre de Lucy.

Même chambre d'hôtel que celle de Leslie, précédemment.

Lucy regarde sa montre. (DH, p. 91)

Ainsi l'auto-référence permet de construire un film fluide auquel l'alternance entre la réalité fictive $\left(\mathrm{W}_{\mathrm{H}}\right)$ et la fiction romanesque confère un rythme. La référence au personnage de Harry est ainsi mise en évidence tout en permettant aux spectateurs de suivre sans trop de heurts plusieurs histoires à la fois.

\section{Reconstructing Woody Allen}

Dans Deconstructing Harry, Woody Allen construit une variété de mondes possibles qui transgressent certains principes logiques auxquels le lecteur est soumis dans $\mathrm{W}_{\mathrm{L}}$. La coexistence des différents niveaux n'est cependant pas incompatible avec une intrigue que le lecteur peut suivre avec intérêt, même si l'auto-référence y est parfois si marquée que le lecteur peut confondre le réel de Harry et son œuvre romanesque. C'est d'ailleurs pourquoi l'étude du scénario s'avère pertinente dans une perspective théorique comme celle de la racontabilité, ainsi définie par Marie-Laure Ryan: "La racontabilité dépend de la diversification des mondes possibles au sein de l'univers narratif, la complexité d'une intrigue dépend d'un système sous-jacent de récits virtuels enchâssés ${ }^{20}$.»

Les trois niveaux de fiction élaborés dans Deconstructing Harry diffèrent quant à leurs macro-contraintes extensionnelles modales. Woody Allen y exploite plus particulièrement les possibilités qu'offre la manipulation des modalités aléthiques et épistémiques. J'ai choisi parmi une multitude de possibles pour illustrer la richesse structurale, narrative et sémantique du film. De plus, l'entrecroisement des niveaux fictionnels provoque la réunion de personnages, de laquelle émerge une critique humoristique de la fiction $^{21}$. Car, de quelque niveau qu'ils soient, il appert que les

20. Marie-Laure Ryan, Possible Worlds, Artificial Intelligence, and Narrative Theory, Bloomington, Indiana University Press, 1991.

21. «FAY: J'aime ton œuvre. J'aime ton imagination. J'aime...

HARRY: Mais, ceci n'est pas un livre, tu comprends. Nous ne sommes pas des personnages dans une œuvre de fiction" (DH, p. 93).

«HARRY: J'écris bien, mais c'est tout à fait autre chose, parce que tu vois, je peux manipuler les personnages et les intrigues. 
personnages possèdent une conscience de leur condition et de leurs origines qui leur procure tous les outils et l'indépendance nécessaires pour créer eux-mêmes des mondes possibles dans lesquels évoluent des agents, dont on se doute bien qu'ils héritent de la schizophrénie de leurs créateurs.

RICHARD: Oui, voilà, tu crées ton propre univers, c'est ça! Mais il est bien meilleur que le monde que nous avons, je crois» (DH, p. 179). 